\title{
Conductive hearing loss in children with autism
}

\author{
Zielinski Rafal
}

Received: 20 January 2013 / Accepted: 19 February 2013 /Published online: 10 March 2013

(C) The Author(s) 2013. This article is published with open access at Springerlink.com

\begin{abstract}
Infantile autism is a serious comprehensive developmental disorder. The diagnosis of hearing loss or its exclusion, which often suggests suspected autism, is very important for early ENT, psychotherapy, and psychiatric treatment. One hundred children diagnosed with autism aged from 3 to 18 years, with a median age of 5 years, were evaluated. The control group of healthy children consisted of 100 children, aged from 3 to 18 years, with a median age of 6 years. Anamnesis and physical examination, including pediatric assessment and otoscopic examination, were carried out on children in both groups. Each child underwent bilateral otoacoustic emission examination in the $0.7,1,2$, and $4 \mathrm{kHz}$ bands and impedance audiometry examination. The data obtained were subjected to a basic statistical assessment. $\mathrm{Chi}^{2}$ Pearson's test was used to compare results of tests in both groups. The absence of otoacoustic emission for the 1 and $2 \mathrm{kHz}$ bands was significantly more frequent in the group of autistic children than in the control group. Furthermore, types B and C2 tympanometric curves were significantly more common in the group of autistic children than in the group of healthy children.
\end{abstract}

Keywords Autism $\cdot$ Children $\cdot$ Conductive hearing loss

\section{Introduction}

Autism is a serious pervasive developmental disorder. Its typical features are not only problems associated with communicating with people and forming social relationships but also integration of sensual perception. It is typical, that autistic children avoid social interactions and they are indifferent to people, even to those who are close relatives. They

\section{Z. Rafal $(\bowtie)$}

Department of Pediatric Otorhinolaryngology,

Medical University of Lodz, ul. Sporna 36/50,

91-738 Lodz, Poland

e-mail: rafal.zielinski@umed.lodz.pl also avoid stimuli triggering a willingness to maintain emotional and verbal contact.

The prevalence for children with autism amounts to $1 / 160$, and it is more common among boys than girls by a ratio of $4: 1$; however, the etiology of autism is most probably multifactorial and is not entirely elucidated. Nowadays, psychosocial factors have been replaced with views about dysfunction of the central nervous system, excessive sensitivity to stimuli and difficulties in their integration. However, as specific autistic changes in the central nervous system have not been found, biological factors are still believed to interact with psychological factors, although their contributions may differ in different autism cases.

The diagnostic criteria of autism were approved by the WHO in 1992. The disease starts before the end of the third year of life and disruptions in speech development are the cause of significant cognitive limitations and difficulties in achieving school readiness. Other characteristic features of autism are hypersensitivity to sensory stimuli, psychomotor hyperreactivity or excessive calmness, poor physical coordination despite proper motor activity, aggression and especially often self-aggression, disorders of multisensory integration, and sleep disorders. The prognosis regarding the cure is poor.

The treatment of children with autism should take into consideration the needs of the child and his/her family. The most common approach is improvement of behavior through behavioral therapy, therapy of disordered sensor integration, speech therapy, and symptomatic pharmacological treatment. However, due to the nature of the condition, the child's caregivers often seek help from a number of different specialists, and as a consequence, the diagnosis of the disease often comes very late. The care of autistic children is also very demanding since along with behavioral and psychiatric conditions, there are other medical issues, that are in focus of many medical specialities [8].

In the age of widespread program of hearing loss screening among newborns, perceptive deafness can be detected before the age of 6 months and is offered until the end of the 
first year of life. The diagnosis of early hearing loss or its exclusion, which often suggests suspected autism, is very important for early hearing aid application, psychotherapy and psychiatric treatment. So it can be said that a program of early hearing loss detection makes a considerable contribution to the early detection of psychiatric disorders among children. Auditory brainstem response testing in such a group of children is difficult to assess because of the existence of many artifacts [6]. To further complicate matters, a frequent problem, which occurs during the school and preschool periods, is conductive hearing loss, as well as mixed hearing loss, which is often unnoticed even among healthy children [5].

In the group of children with emotional and psychiatric/ psychological problems, hearing loss may be masked by the child's behavior and remains unnoticed. Hearing impairment among children with developmental disorders has a special significance: it can cause even more serious disorders connected with the people around them, which has a significant impact on the child's development, rehabilitation, and psychiatric/psychological treatment [9]. The problem is also present in children with unilateral hearing loss as it affects hearing in noise and sound source localization [2].

As subjective tests in autistic children are particularly challenging to perform due to the difficulty in making contact with them, using objective methods would seem to be the most suitable approach to assess hearing loss in the population of school and preschool children with psychological problems [11].

\section{Material and methods}

One hundred children diagnosed with autism, confirmed by psychological, psychiatric, and neurological examination performed on the basis of WHO criteria, took part in the study. The control group consisted of 100 children without diagnosed disorders, confirmed by psychological, psychiatric, and neurological examination.

Anamnesis and physical examinations, including pediatric assessment and otoscopy, were carried out on children in both groups; the aim of which were to exclude pathology of the external auditory canal, which can have an influence on results of examinations assessing the condition of the middle ear. The gender and age of a child were assessed. Each child underwent a series of tests: bilateral otoacoustic emission test in the $0.7,1,2$, and $4 \mathrm{kHz}$ bands and impedance audiometry examination with tympanometric curve assessment. The data were subjected to basic statistical assessment. $\mathrm{Chi}^{2}$ Pearson's test was also used to compare results of tests in healthy and autistic children population at significance level $\alpha=0.05$.
66

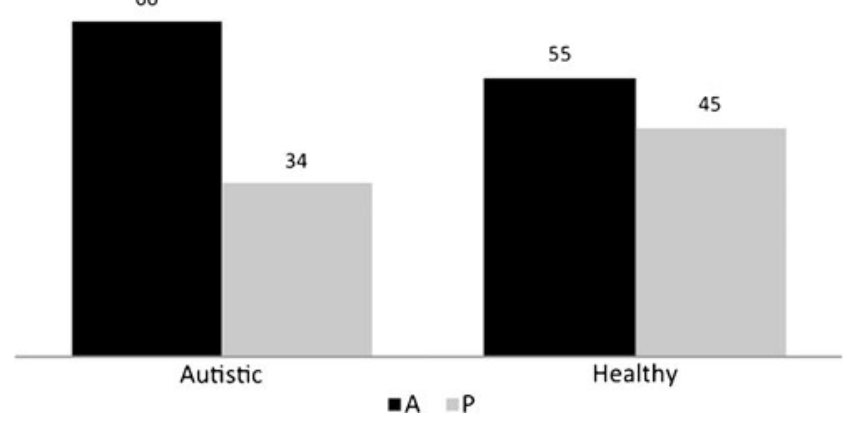

Fig. 1 Otoacoustic emission in the $0.7 \mathrm{kHz}$ band in autistic and healthy children. $A$ absent in one or two ears, $P$ present in two ears

\section{Results}

The gender compositions of the study groups were as follows: in the autistic group, there were 81 boys and 19 girls, while in the control group, 51 boys and 49 girls. These numbers reflect the proportions of genders in populations of healthy children, where the number of boys equals the number of girls, and autistic children, where boys are in the majority [1]. Children in both groups were aged from 3 to 18 years. The median age of the children in the autistic group was 5 years. The median age of children in control group was 6 years.

The tests consisted of bilateral otoacoustic emission in the $0.7,1,2$, and $4 \mathrm{kHz}$ bands for the right and left ear and bilateral impedance audiometry examination with tympanometric curve assessment.

With the $0.7-\mathrm{kHz}$ frequency, otoacoustic emission was present in both ears in 34 autistic children and in 45 healthy children. It was not present in both or one ear in 66 autistic children and in 55 healthy children, respectively. The difference in numbers of positive results [otoacoustic emission (OAE) $0.7 \mathrm{kHz}$ present] tested with $\mathrm{Chi}^{2}$ Pearson's test was not significant, $\chi^{2}=2.53, p=0.1$ (Fig. 1).

With the $1-\mathrm{kHz}$ frequency, otoacoustic emission was present in both ears in 42 autistic children and in 96 healthy children. It was not present in both or one ear in 58 autistic children and in four healthy children. The difference in

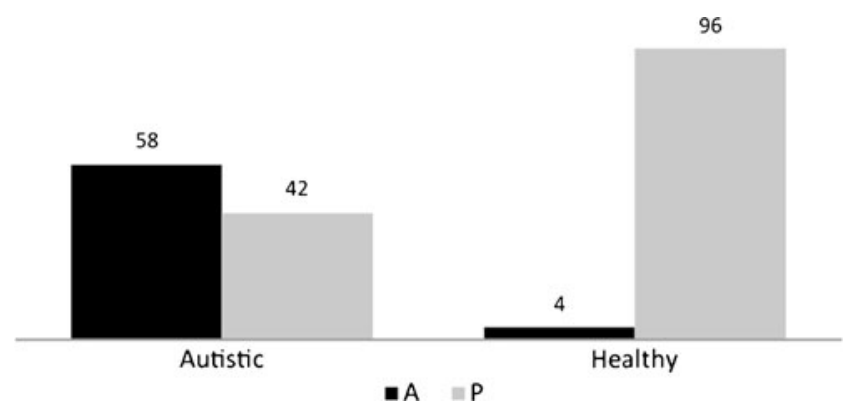

Fig. 2 Otoacoustic emission in the $1 \mathrm{kHz}$ band in autistic and healthy children. $A$ absent in one or two ears, $P$ present in two ears 


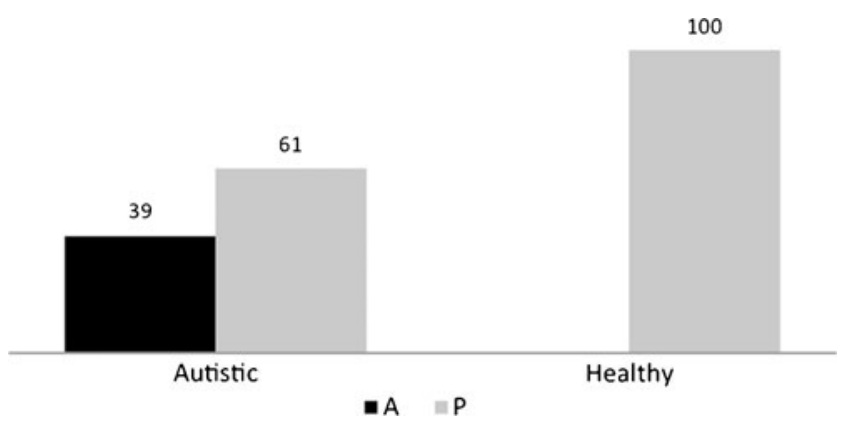

Fig. 3 Otoacoustic emission in the $2 \mathrm{kHz}$ band in autistic and healthy children. $A$ absent in one or two ears, $P$ present in two ears

numbers of positive results (OAE $1 \mathrm{kHz}$ present) tested with $\mathrm{Chi}^{2}$ Pearson's test was significant, $\chi^{2}=68.16, p=0.0001$ (Fig. 2).

With the 2-kHz frequency, otoacoustic emission was present in both ears in 61 autistic children and in 100 healthy children. It was not present in both or one ear in 39 autistic children and in no healthy children. The difference in numbers of positive results (OAE $2 \mathrm{kHz}$ present) tested with $\mathrm{Chi}^{2}$ Pearson's test was significant, $\chi^{2}=48.44$, $p=0.0001$ (Fig. 3).

With the $4-\mathrm{kHz}$ frequency, otoacoustic emission was present in both ears in 60 autistic children and in 64 healthy children. It was not present in both or one ear in 40 autistic children and in 36 healthy children. The difference in numbers of positive results (OAE $4 \mathrm{kHz}$ present) tested with $\mathrm{Chi}^{2}$ Pearson's test was not significant, $\chi^{2}=0.34, p=0.56$ (Fig. 4).

Tympanometric curves associated with a healthy middle ear on both sides (namely A and C1 curves) were described in 75 autistic children and in 92 healthy children. Tympanometric curves associated with dysfunctional middle ear (namely B and C2 curves) on both or one side were described in 25 autistic children and in eight healthy children, respectively. The difference in numbers of pathological results ( $\mathrm{B}$ or $\mathrm{C} 2$ curve present in one or two ears) tested with $\mathrm{Chi}^{2}$ Pearson's test was significant, $\chi^{2}=10.49$, $p=0.001$ (Fig. 5).

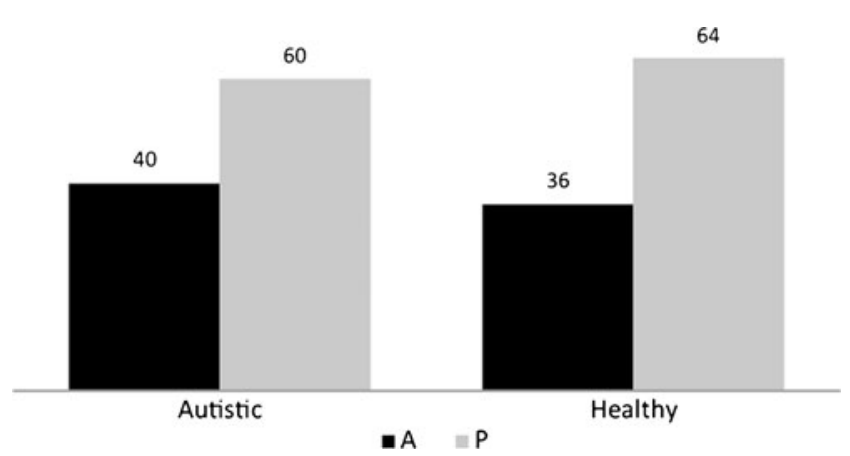

Fig. 4 Otoacoustic emission in the $4 \mathrm{kHz}$ band in autistic and healthy children. $A$ absent in one or two ears, $P$ present in two ears



Fig. 5 Tympanometric curves in autistic and healthy children. $A C 1 \mathrm{~A}$ or $\mathrm{C} 1$ curves present in two ears, $B C 2 \mathrm{~B}$ or $\mathrm{C} 2$ present in one or two ears

Only six children in the group of autistic children were under the care of an otolaryngologist two of whom displayed abnormal examinations in objective tests. In the control group, 17 children were receiving care from an otolaryngologist despite lack of ENT complaints. The difference in numbers of children under the care of otolaryngologist (otolaryngologist care present) tested with $\mathrm{Chi}^{2}$ Pearson's test was significant, $\chi^{2}=5.94, p=0.01$ (Fig. 6).

\section{Discussion}

Conductive hearing loss in pre- and early school children is most commonly caused by dysfunction of the middle ear, which is caused by disorders in the ventilation of the tympanic cavity [10]. An early diagnosis of Eustachian tube dysfunction and its results, such as chronic otitis media with effusion and chronic atelectatic otitis media, plays an important role in stopping the progression of disease and restoring hearing, which are indispensable in the proper psychosocial development of a young child [7]. It is also exceedingly important in the group of children with the pervasive developmental disorder that is autism. Bilateral hearing, development and consolidation of proper speech in these children are not only a necessary element of development but also serve as a rehabilitation tool.

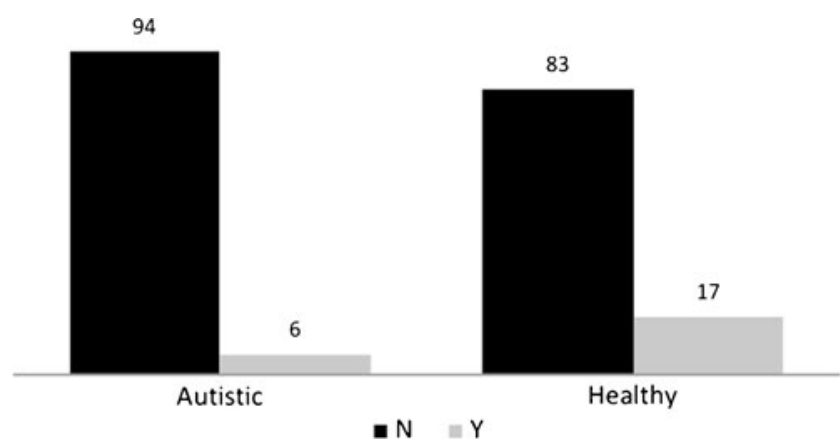

Fig. 6 Otolaryngologist care in autistic and healthy children. $N$ no otolaryngological care, $Y$ under otolaryngological care 
Tests such as otoacoustic emission and impedance audiometry are invaluable tools in the screening of middle ear problems in children. They have particular importance in an uncooperative group of children, a group to which autistic children undoubtedly belong. Otoacoustic emission examination is widely used as a tool for screening in hearing loss. The absence of otoacoustic emission is considered to be typical of conductive hearing loss in the course of otitis media with effusion [3, 12]. In this study, the absence of otoacoustic emission for the 1 and $2 \mathrm{kHz}$ bands was significantly more frequent in the group of autistic children than in the control group. The absence of otoacoustic emission for the above bands is practically important as the bands are situated within voice frequencies.

The impedance audiometry examination is most commonly used as an objective examination allowing the assessment of middle ear functions. The assessment of the tympanometric curves makes it possible to recognize serious middle ear ventilation problem. The presence of types B and $\mathrm{C} 2$ tympanometric curves is most frequently observed in chronic otitis media with effusion, which is a common cause of conductive hearing loss in children [4]. The curves were significantly more commonly observed in the group of autistic children than in the group of healthy children.

\section{Conclusions}

1. The examination results suggest that conductive hearing loss is a frequent problem in children with autism. It is often masked by autistic behavior and remains unnoticed.

2. It is worth noticing that autistic children are not provided with proper otolaryngological care. Compulsory periodic hearing evaluation should be introduced in the group of children with comprehensive developmental disorders like autism.

Acknowledgments The author would like to appreciate the help from members of Audiology Team in the Department of Pediatric Otorhinolaryngology, Medical University of Lodz.
Conflict of interest The author discloses any financial and personal relationships with other people or organizations that could inappropriately influence (bias) his work.

Open Access This article is distributed under the terms of the Creative Commons Attribution License which permits any use, distribution, and reproduction in any medium, provided the original author(s) and the source are credited.

\section{References}

1. Beacher FD, Radulescu E, Minati L, Baron-Cohen S, Lombardo MV, Lai MC, Walker A, Howard D, Gray MA, Harrison NA, Critchley HD (2012) Sex differences and autism: brain function during verbal fluency and mental rotation. PLoS One 7(6):e38355. doi:10.1371/journal.pone.0038355

2. Briggs L, Davidson L, Lieu JE (2011) Outcomes of conventional amplification for pediatric unilateral hearing loss. Ann Otol Rhinol Laryngol 120(7):448-454

3. Dragicevic D, Vlaski L, Komazec Z, Jovic RM (2010) Transient evoked otoacoustic emissions in young children with otitis media with effusion before and after surgery. Auris Nasus Larynx 37(3):281-285. doi:10.1016/j.anl.2009.09.004

4. Kemaloglu YK, Sener T, Beder L, Bayazit Y, Goksu N (1999) Predictive value of acoustic reflectometry (angle and reflectivity) and tympanometry. Int J Pediatr Otorhinolaryngol 48(2):137-142

5. Kon K, Inagaki M, Kaga M, Sasaki M, Hanaoka S (2000) Otoacoustic emission in patients with neurological disorders who have auditory brainstem response abnormality. Brain Dev 22(5):327-335

6. Matas CG, Goncalves IC, Magliaro FC (2009) Audiologic and electrophysiologic evaluation in children with psychiatric disorders. Braz J Otorhinolaryngol 75(1):130-138

7. Obrębowski A, Obrębowska Z (2009) Wpływ przewlekłego wysiekowego zapalenia ucha środkowego na rozwój mowy u dzieci. Otolaryngologia 8(4):159-162

8. Olivie H (2012) The medical care of children with autism. Eur J Pediatr 171(5):741-749. doi:10.1007/s00431-011-1669-1

9. Rosenhall U, Nordin V, Sandstrom M, Ahlsen G, Gillberg C (1999) Autism and hearing loss. J Autism Dev Disord 29(5):349-357

10. Takahashi H, Hayashi M, Sato H, Honjo I (1989) Primary deficits in eustachian tube function in patients with otitis media with effusion. Arch Otolaryngol Head Neck Surg 115(5):581-584

11. Tas A, Yagiz R, Tas M, Esme M, Uzun C, Karasalihoglu AR (2007) Evaluation of hearing in children with autism by using TEOAE and ABR. Autism 11(1):73-79. doi:10.1177/1362361307070908

12. Zhao F, Wada H, Koike T, Ohyama K, Kawase T, Stephens D (2003) Transient evoked otoacoustic emissions in patients with middle ear disorders. Int J Audiol 42(3):117-131 